\title{
Synthesis and characterization of textural and thermal properties of polymer monoliths
}

\author{
M. Maciejewska
}

Received: 20 November 2014 / Accepted: 3 February 2015/Published online: 29 March 2015

(c) The Author(s) 2015. This article is published with open access at Springerlink.com

\begin{abstract}
Polymer monoliths with highly developed porous structure have been successfully synthesized by polymerization of 1-vinyl-2-pyrrolidone with trimethylolpropane trimethacrylate and divinylbenzene. During the copolymerization, the mixtures of toluene/n-dodecane and toluene/decan-1-ol were used as pore-forming diluents. The thermodynamic quality of the porogen mixtures strongly influences the process of the pore formation. By changing the ratio of non-solvating and solvating diluents, monoliths with surface area from 57 to $650 \mathrm{~m}^{2} \mathrm{~g}^{-1}$ and pore diameters from 4 to $51 \mathrm{~nm}$ were prepared. Thermal properties of the obtained materials were investigated by the means of thermogravimetry and differential scanning calorimetry. It was found that they do not depend on the diluent composition but are determined by the type of cross-linker used in the synthesis.
\end{abstract}

Keywords Polymers synthesis · Porous structure · 1-Vinyl-2-pyrrolidone · Thermal properties .

Thermogravimetry · Differential scanning calorimetry

\section{Introduction}

One of the significant trends in modern chromatography is searching for new materials as adsorbents to improve the mass transfer, allow fast separation and provide column stability in the whole range of $\mathrm{pH}$. The packing based on porous polymers can meet these requirements. Additionally, they provide a huge variety of functional groups

M. Maciejewska $(\square)$

Faculty of Chemistry, Maria Curie-Skłodowska University, pl. M. Curie-Skłodowskiej 3, 20-031 Lublin, Poland

e-mail: mmacieje@umcs.pl available on the surface [1-8]. Over the past years, the major attention of scientists and producers was focused on particle-packed columns with smaller and smaller microspheres. Nowadays, porous polymeric monoliths become more popular [9-15]. Comparing with particle-packed columns, monolithic columns possess several considerable advantages: The final product has the desired geometry and there is no waste; almost any material can be used including water soluble monomers, e.g., 1-vinyl-2-pyrrolidone (VP). Despite its valuable properties like nontoxicity, biocompatibility and good complexing properties for ionic or p-electron system VP can be hardly used in suspension polymerization because of the solubility of the monomer in an aqueous continuous phase. The attempts to obtain copolymers of 1-vinyl-2-pyrrolidone with different cross-linkers by suspension or suspension-emulsion polymerization result in limited amount of VP incorporated in the copolymer matrix [16-21]. This limitation can be overcome to a great extent by the synthesis of monoliths. Additionally, in the case of direct synthesis in the chromatographic columns, no tedious sieve and packing operations are required. Therefore, development of the monolithic columns with conventional analytical dimensions for the separation of small molecules is desirable.

Application of the obtained monoliths as HPLC packing requires a sufficiently large specific surface area that provides interacting sites. The most substantial contribution to the overall surface area comes from the micropores. However, in terms of HPLC measurements, micropores lead to reduction in mass transfer and peak broadening. As a result, pores with sizes smaller than $2 \mathrm{~nm}$ are undesirable in the internal structure of the monoliths. Simultaneously, meso- $(2-50 \mathrm{~nm})$ and macropores $(>50 \mathrm{~nm})$ should be highly interconnected. Considerable pore connectivity facilitates the mass transfer kinetics and allows the mobile 
Fig. 1 Schema of the synthesis of the porous monoliths<smiles>C=C(C)C(=O)OCC(CC)(COC(=O)C(=C)C)COC(=O)C(=C)C</smiles><smiles>C=CN1CCCC1=O</smiles>

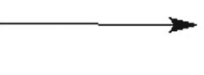

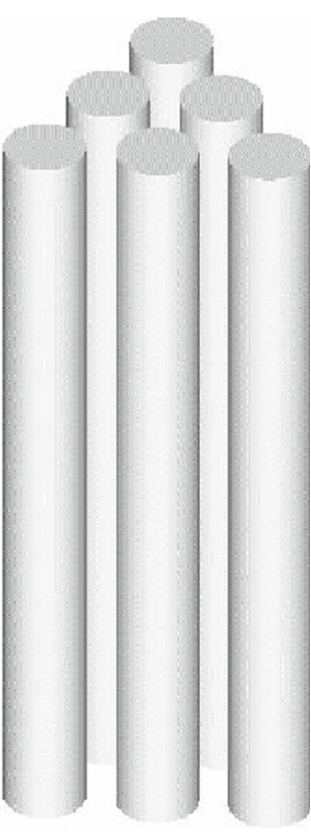

phase to flow through the material at a reasonably low pressure. The required porous structure can be obtained using proper porogenic solvents (porogens). The porogen controls the porous structure of the monoliths by the process of solvation of the polymer chains in the reaction medium during the early stages of the polymerization. The choice of proper solvent is of paramount importance in the preparation of polymer monoliths [22, 23].

The aim of this paper was to synthesize and characterize textural and thermal properties of polymer monoliths with pyrrolidone functionalities and optimal morphology for HPLC applications. To obtain this goal, copolymers of VP with divinylbenzene (DVB) and trimethylolpropane trimethacrylate (TRIM) were directly synthesized in the columns for high-performance liquid chromatography. Textural and thermal properties were investigated in details.

\section{Experimental}

\section{Chemicals}

1-vinyl-2-pyrrolidone (Fluka AG, Buchs, Switzerland), divinylbenzene (Merck, Darmstadt, Germany) and trimethylolpropane trimethacrylate (Sigma-Aldrich, Steinheim, Germany) were washed with $5 \%$ aqueous sodium hydroxide in order to remove inhibitors. Toluene, $n$-dodecane, decan-1-ol (reagent grade) were from Fluka, whereas methanol (HPLC grade) was from Merck.
Table 1 Solubility parameters of the used solvent and solvent mixtures

\begin{tabular}{ll}
\hline Solvent & Solubility parameter $\delta / \mathrm{MPa}^{1 / 2}$ \\
\hline Toluene & 18.2 \\
$n$-Dodecane & 16.2 \\
Decan-1-ol & 17.6 \\
Toluene : $n$-dodecane $5.6: 1 \mathrm{v} / \mathrm{v}$ & 17.9 \\
Toluene : $n$-dodecane $1: 1 \mathrm{v} / \mathrm{v}$ & 17.2 \\
Toluene : $n$-dodecane $1: 5.6 \mathrm{v} / \mathrm{v}$ & 16.5 \\
Toluene $:$ decan-1-ol $5.6: 1 \mathrm{v} / \mathrm{v}$ & 18.1 \\
Toluene : decan-1-ol $1: 1 \mathrm{v} / \mathrm{v}$ & 17.9 \\
Toluene : decan-1-ol $1: 5.6 \mathrm{v} / \mathrm{v}$ & 17.7 \\
\hline
\end{tabular}

\section{Polymerization}

All polymerizations were performed within the HPLC columns $(100 \mathrm{~mm} \times 4.6 \mathrm{~mm})$ provided with a heating jacket. The polymerization mixtures containing functional monomer, cross-linker, porogenic solvents and initiator were prepared and purged with nitrogen for $15 \mathrm{~min}$. The polymerization mixtures were transferred into the stainless steel column. The columns were sealed with PTFE plugs and put into the polymerization vessel in which the polymerization occurred. Thermostated water circulated through the jacket provided the required temperature. The polymerizations were allowed to proceed 

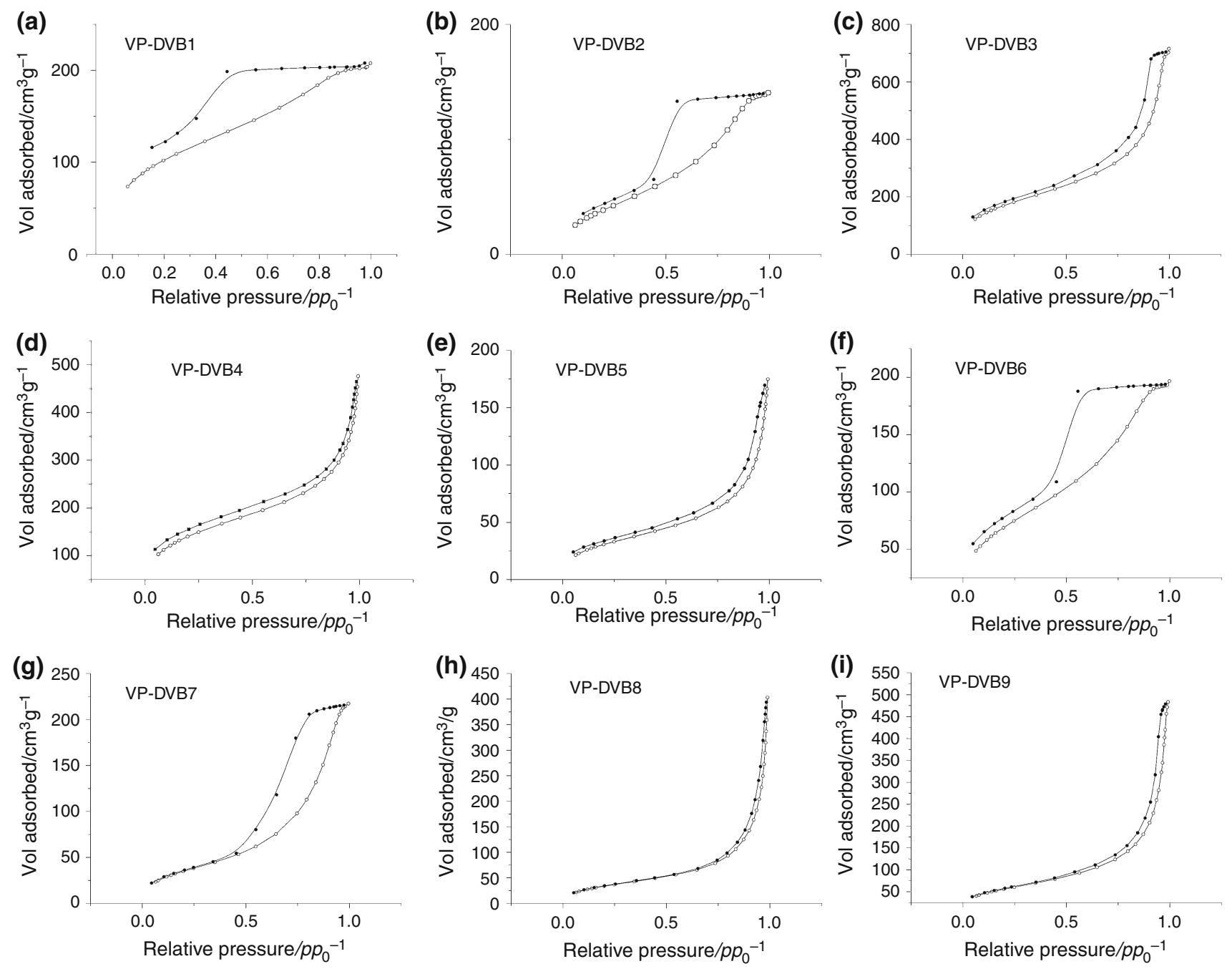

Fig. 2 Nitrogen adsorption (opened circle) and desorption (filled circle) isotherms at $77 \mathrm{~K}$ of VP-DVB monoliths

for $24 \mathrm{~h}$. After the reactions were completed, the plugs were replaced by columns end fittings, and the rods were exhaustively washed with methanol using dual piston high-performance constant pressure pump. Finally, the obtained copolymers were dried under vacuum at $80{ }^{\circ} \mathrm{C}$ for $24 \mathrm{~h}$.

\section{Method of analysis}

The surface of the obtained beads was examined using an atomic LEO 1430 VP numerical scanning electron microscope (Germany) with a countershaft and an energy dispersive X-ray detector.

Elemental analysis of the obtained microspheres was carried out using the Perkin Elmer CHN 2400 apparatus.

Characterization of the porous structure of the monoliths was carried out by the low-temperature nitrogen adsorption-desorption method. Nitrogen adsorption-desorption measurements were made at $77 \mathrm{~K}$ using a volumetric adsorption analyzer ASAP 2405 (Micrometrics Inc., USA). The measurements of the surface properties of the copolymers were preceded by outgassing the samples at $140{ }^{\circ} \mathrm{C}$ for $2 \mathrm{~h}$.

The maximum of PSD was defined as pore diameter in contrast to the average pore diameter calculated as $D_{\mathrm{p}}=4 V_{\mathrm{p}}$ $S_{\mathrm{BET}}$ (on assumption of a cylindrical shape of pores).

The thermal properties of the synthesized monoliths were evaluated on the basis of TG and DSC measurements.

Thermogravimetric analysis of materials was carried out with a Netzsch STA 449 F1 Jupiter thermal analyzer (Germany). The procedure was as follows: About $10 \mathrm{mg}$ of the sample was placed in the TG pan and heated in helium atmosphere at a rate of $10 \mathrm{~K} \mathrm{~min}^{-1}$ up to $1000{ }^{\circ} \mathrm{C}$. The gas flow was $20 \mathrm{~mL} \mathrm{~min}{ }^{-1}$. The initial decomposition temperature (IDT), $T_{20} \%, T_{50} \%$, of mass loss and final decomposition temperature (FDT) were determined. 
(a)

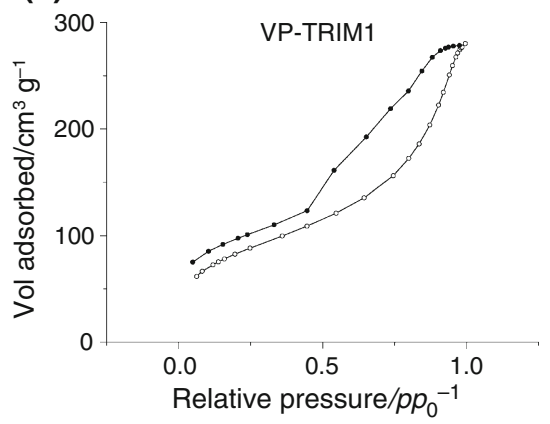

(d)

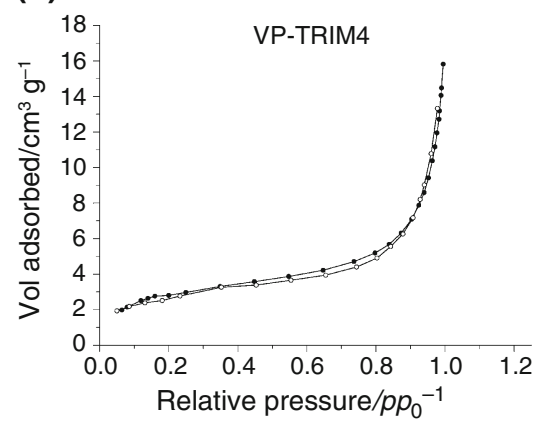

(g)

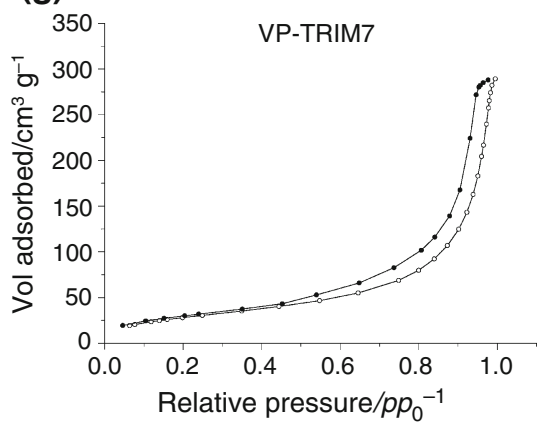

(b)

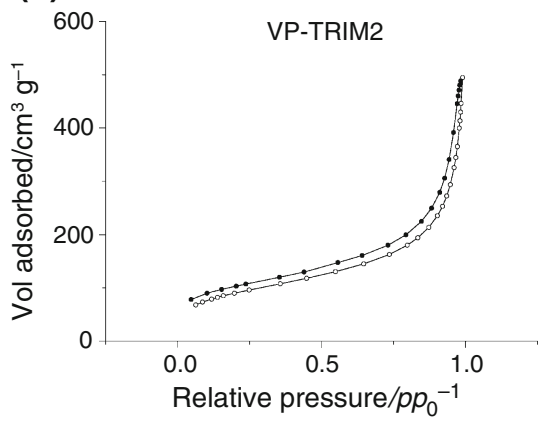

(e)

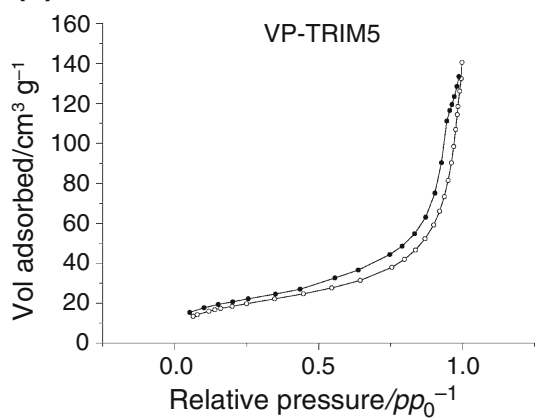

(h)

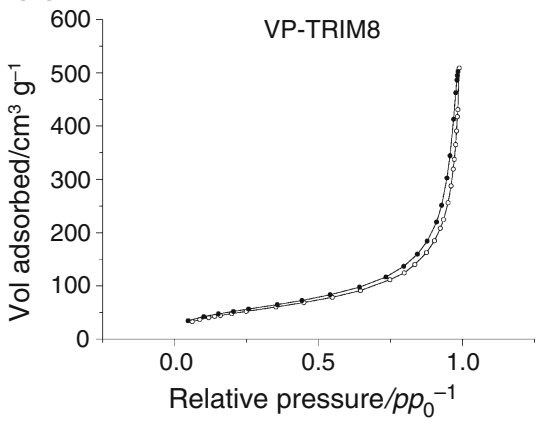

(c)

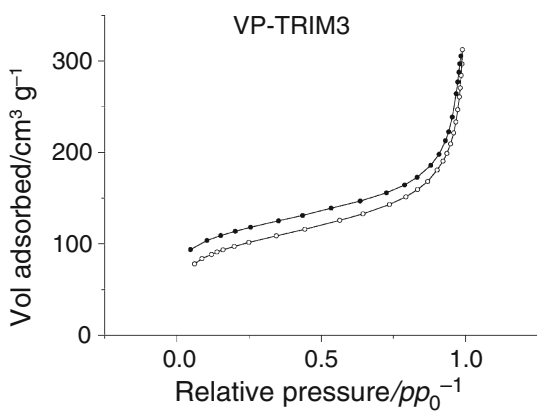

(f)

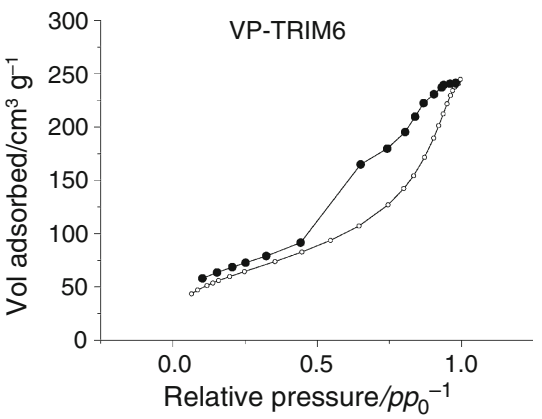

(i)

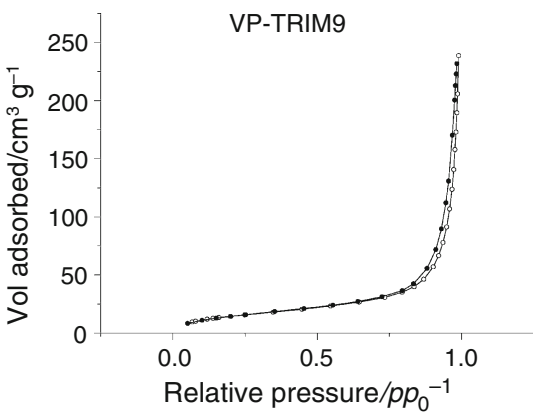

Fig. 3 Nitrogen adsorption (opened circle) and desorption (filled circle) isotherms at $77 \mathrm{~K}$ of VP-TRIM monoliths

Calorimetric measurements were carried out with the Netzsch DSC 204 calorimeter (Germany) operating in the dynamic mode. The dynamic scans were performed at the heating rate of $10 \mathrm{~K} \mathrm{~min}^{-1}$ from room temperature to the maximum of $500{ }^{\circ} \mathrm{C}$ under argon (gas flow $20 \mathrm{~mL} \mathrm{~min}^{-1}$ ) atmosphere. The mass of the sample was $7 \mathrm{mg}$. As a reference, an empty aluminum crucible was used.

\section{Results and discussion}

Polymer monoliths were prepared by free radical crosslinking polymerization. In the synthesis, two different cross-linkers were used DVB and TRIM (Fig. 1). They were responsible for the mechanical and thermal properties of the resulting monoliths. VP was used as functional monomer in order to introduce pyrrolidone units into the polymeric matrix.

To obtain porous rigid material that exhibits considerable porosity, the polymerization mixture must contain large amounts of porogen. In most cases, the porogens are simple organic solvents or their mixtures. The morphology of the polymers is predominantly determined by the phase separation during polymerization. The process of phase separation strongly depends on the solvating power of the used porogen. In the case of high solvating power of the diluent, phase separation occurs late at greater conversion of monomer to polymer. As a result, a network of interconnecting individual microglobules is formed. The network is characterized by high surface area and pore size 
Table 2 Parameters of the porous structure of the monoliths under study

\begin{tabular}{|c|c|c|c|c|c|c|}
\hline \multirow[t]{2}{*}{ Monolith } & \multicolumn{3}{|c|}{ Diluents/mL } & \multirow{2}{*}{$\begin{array}{l}\text { Specific surface } \\
\text { area } S_{\mathrm{BET}} / \mathrm{m}^{2} \mathrm{~g}^{-1}\end{array}$} & \multirow{2}{*}{$\begin{array}{l}\text { Pore } \\
\text { volume } V / \mathrm{cm}^{3} \mathrm{~g}^{-1}\end{array}$} & \multirow{2}{*}{$\begin{array}{l}\text { Pore diameter } \\
D_{\mathrm{BJH}} / \mathrm{nm}\end{array}$} \\
\hline & Toluene & Dodecane & Decan-1-ol & & & \\
\hline VP-TRIM1 & 9 & - & - & 306 & 0.457 & 4 \\
\hline VP-TRIM2 & 7.6 & 1.4 & - & 331 & 0.790 & 39 \\
\hline VP-TRIM3 & 4.5 & 4.5 & - & 347 & 0.435 & 40 \\
\hline VP-TRIM4 & 1.4 & 7.6 & - & 108 & 0.242 & $2.5 / 40$ \\
\hline VP-TRIM5 & - & 9 & - & 69 & 0.208 & 16 \\
\hline VP-TRIM6 & 7.6 & - & 1.4 & 224 & 0.391 & 4 \\
\hline VP-TRIM7 & 4.5 & - & 4.5 & 107 & 0.447 & 25 \\
\hline VP-TRIM8 & 1.4 & - & 7.6 & 184 & 0.790 & 50 \\
\hline VP-TRIM9 & - & - & 9 & 57 & 0.368 & 50 \\
\hline VP-DVB1 & 9 & - & - & 381 & 0.321 & 4 \\
\hline VP-DVB2 & 7.6 & 1.4 & - & 650 & 1.178 & 6 \\
\hline VP-DVB3 & 4.5 & 4.5 & - & 633 & 1.131 & 19 \\
\hline VP-DVB4 & 1.4 & 7.6 & - & 521 & 0.727 & 20 \\
\hline VP-DVB5 & - & 9 & - & 117 & 0.277 & 23 \\
\hline VP-DVB6 & 7.6 & - & 1.4 & 262 & 0.321 & 4 \\
\hline VP-DVB7 & 4.5 & - & 4.5 & 136 & 0.336 & 6 \\
\hline VP-DVB8 & 1.4 & - & 7.6 & 134 & 0.421 & 49 \\
\hline VP-DVB9 & - & - & 9 & 85 & 0.473 & 51 \\
\hline
\end{tabular}

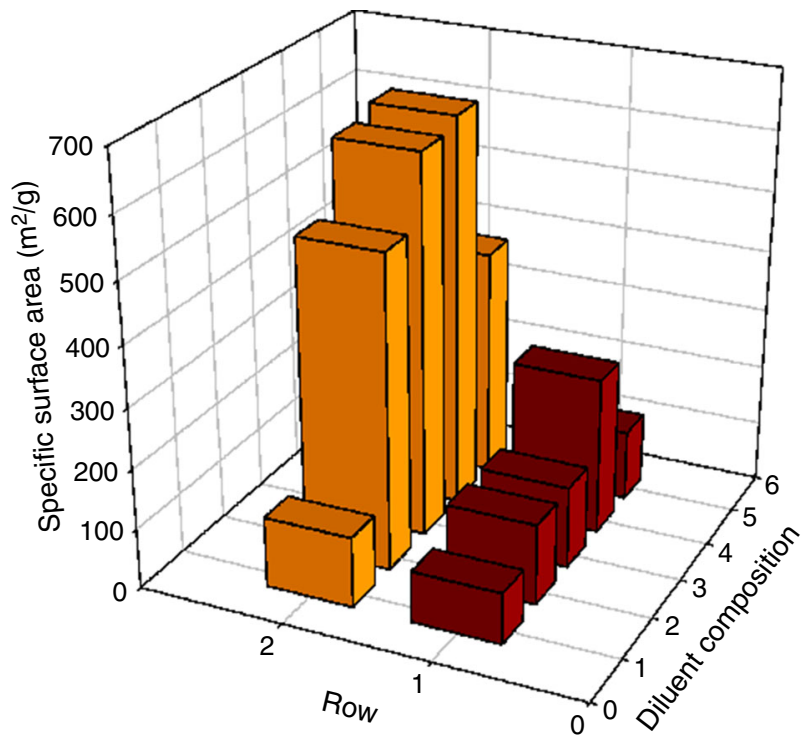

Fig. 4 Dependence of the toluene content in the mixture with decan1-ol (row 1) and dodecane (row 2) on the specific surface area for VPDVB copolymer. 1-0 \% toluene, 2-15\% toluene; $3-50 \%$ toluene, 4-85\% toluene, $100 \%$ toluene

distribution with a maximum in the region of micro- to mesopore. Application of non-solvating diluents leads to early phase separation at lower conversion. This process provokes not only aggregation of microglobules but also in

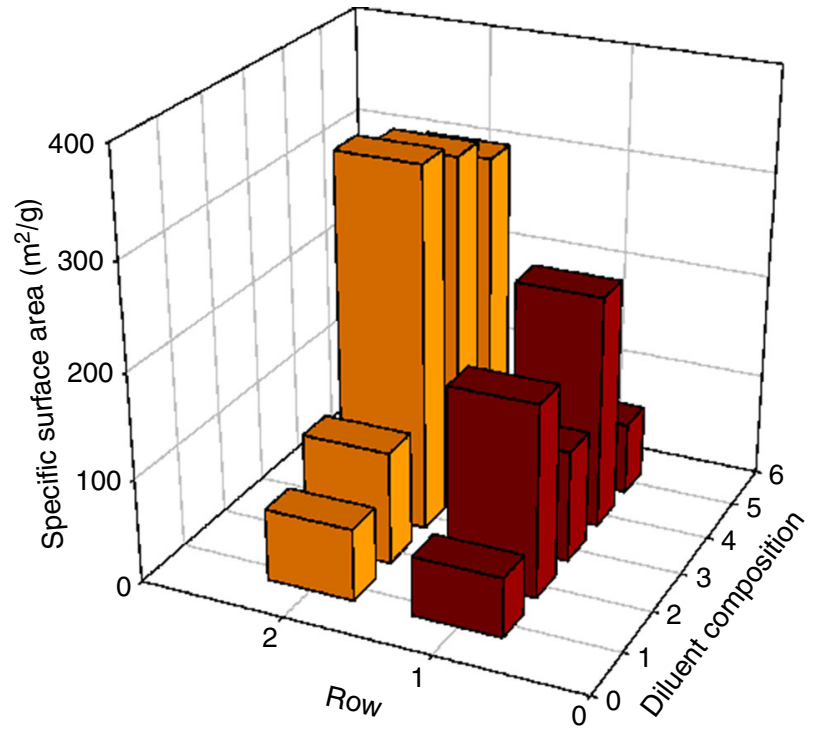

Fig. 5 Dependence of the toluene content in the mixture with decan1-ol (row 1) and dodecane (row 2) on the specific surface area for VPTRIM copolymer. 1-0 \% toluene, 2-15\% toluene; 3-50\% toluene, 4-85\% toluene, $100 \%$ toluene

filling of small pores to form polymer network with low surface area. The solvating power of the diluents can be evaluated on the basis of Hildebrand's solubility parameters $\delta$. In the synthesis of the porous monoliths, three 


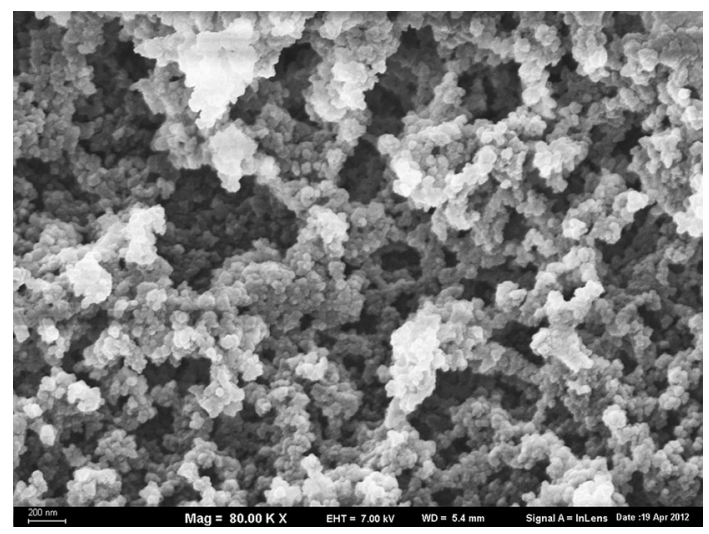

Fig. 6 SEM image of the internal structure of the monolith obtained in the presence of pure toluene (VP-DVB1)

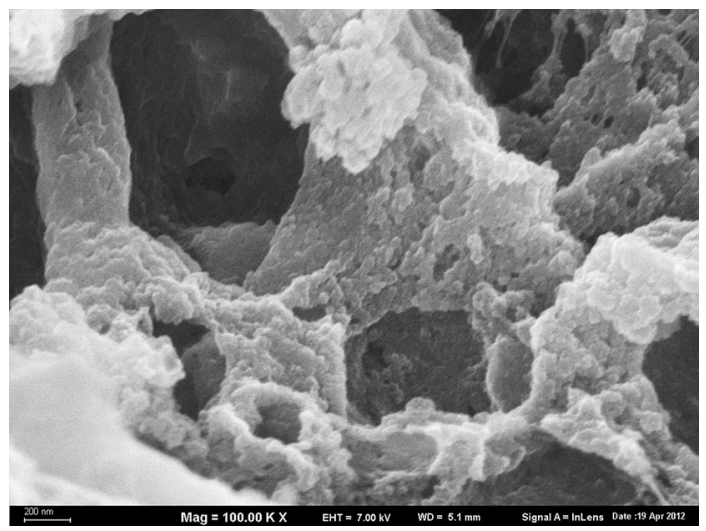

Fig. 7 SEM image of the internal structure of the monolith obtained in the presence of pure decan-1-ol (VP-DVB9)

different solvents and their mixtures were applied. Table 1 contains solubility parameters for all the used solvent and solvent mixtures. The values of solubility parameters of the pure solvents were obtained from literature [24], whereas for the mixtures of solvents were calculated from the relationship of Lloyd et al. [25]. This relationship Eq. (1) relates the solubility parameter for a mixture of solvents to that of the individual solvents and their volume fraction $\phi$ :

$\delta^{2}=\phi_{1} \delta_{1}^{2}+\phi_{2} \delta_{2}^{2}$

where $\delta_{1}$ solubility parameter of the first solvent, $\phi_{1}$ volume fraction of the first solvent, $\delta_{2}$ solubility parameter of the second solvent, $\phi_{2}$ volume fraction of the first solvent.

Toluene is thermodynamically good solvent for the preparation of VP-based monoliths crosslined with TRIM as well as with DVB. These systems are characterized by a small difference between the Hildebrand's solubility parameters for both diluent and porogen. $n$-dodecane and decan-1-ol act as non-solvating diluents. Varying the ratio between these solvents thus alters the overall thermodynamic quality of the porogen and consequently the porous structure of the copolymers under study. Information about their internal structure was drawn on the basis of the adsorption-desorption isotherms of nitrogen on the investigated samples (Figs. 2, 3). According to the IUPAC, they can be classified as type IV that characterizes mesoporous adsorbents [26]. The isotherms of copolymers obtained in the presence of pure toluene (for both types of copolymers) and in solvents mixtures where toluene prevails (for VP-DVB copolymers) show hysteresis loops that can be attributed to capillary condensation. Hysteresis loops may exhibit different shapes. As regards the discussed samples, the hysteresis loops are broad and can be classified as $\mathrm{H} 2$ type. Type $\mathrm{H} 2$ can be found in many porous adsorbent, and this kind of loop occurs when there is a difference in mechanism between condensation and evaporation. This phenomenon takes place in pores with narrow necks and wide bodies (ink bottle shape) or when the porous material has an interconnected pore network. Along with the increasing amount of non-solvating diluent into solvent mixture, the adsorption and desorption branches of isotherm start to merge (Table 1).

The specific surface areas of the investigated copolymers were calculated by the Brunauer-Emmet-Teller (BET) method for the adsorption data in the range of the relative pressure of $p / p_{\mathrm{o}} 0.05-0.25$. Table 2 indicates a close relationship between the composition of the porogenic solvent and the specific surface area. The higher the toluene/decan-1-ol ratio, the higher the surface area. In case of thermodynamically good solvents, phase separation occurs later. Therefore, the concentration of monomer within the nuclei is relatively low, and the attraction between individual nuclei, resulting in coalescence is limited. This leads to smaller microglobules and consequently to higher surface area. In the case of non-solvating diluents, the microglobules are grown together, their individuality is almost lost, and the porosity is lower. As a result, a huge drop in the value of specific surface area is observed. Its value does not exceed $100 \mathrm{~m}^{2} \mathrm{~g}^{-1}$.

However, the effect of mixed porogens is not always a linear interpolation between those encountered for pure solvents. In the case of toluene/decanol mixture, the value of surface area increases to a certain volume ratio of solvent (1: $1 \mathrm{v} / \mathrm{v}$ for the VP-TRIM copolymers and 5.6:1 for the VP-DVB copolymers) and then decreases. Similar effect was observed for analogical copolymers obtained in the form of microspheres [19]. The influence of diluent composition on the value of the specific surface area is graphically presented in Figs. 4 and 5.

The changes in porous structure of the monoliths can be also observed using scanning electron microscopy. 


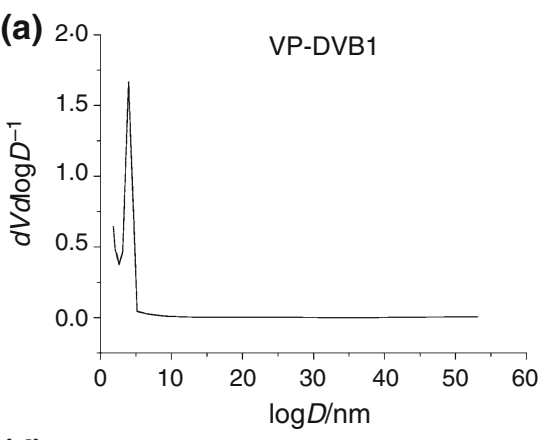

(d)

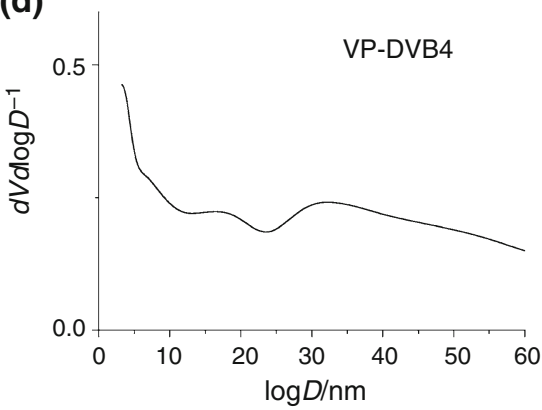

(g)

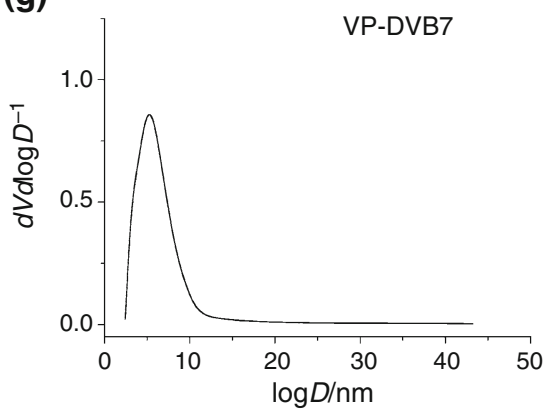

(b)

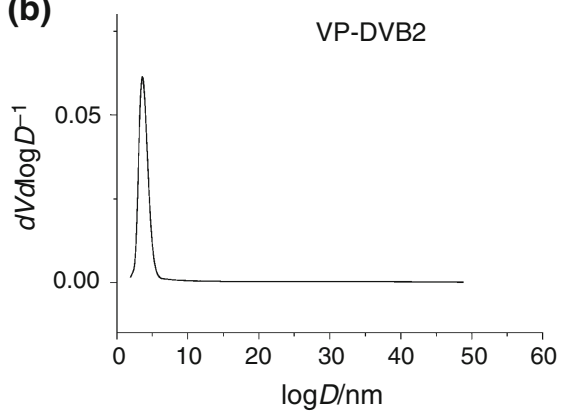

(e)

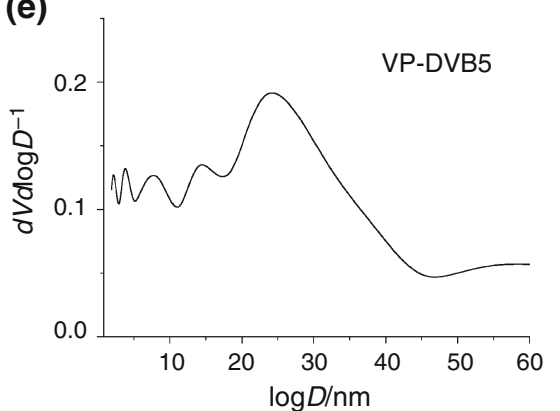

(h)

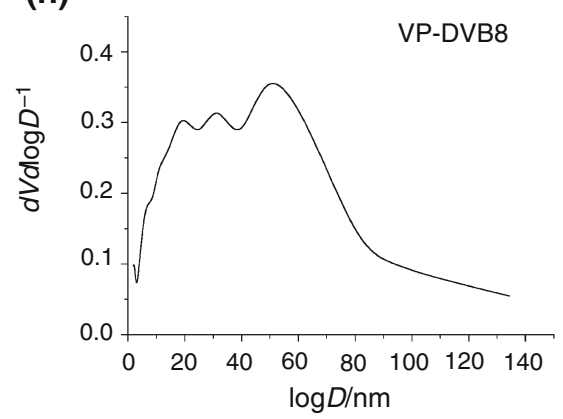

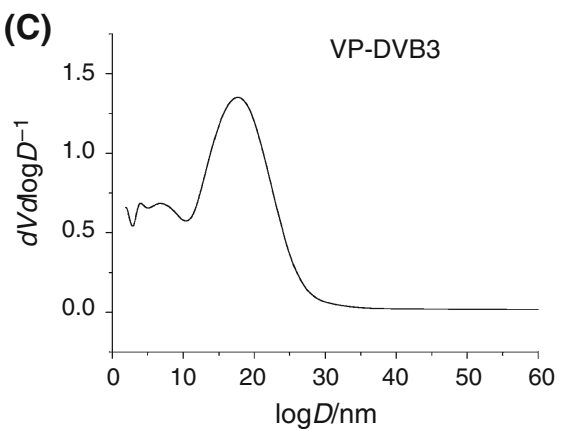

(f)

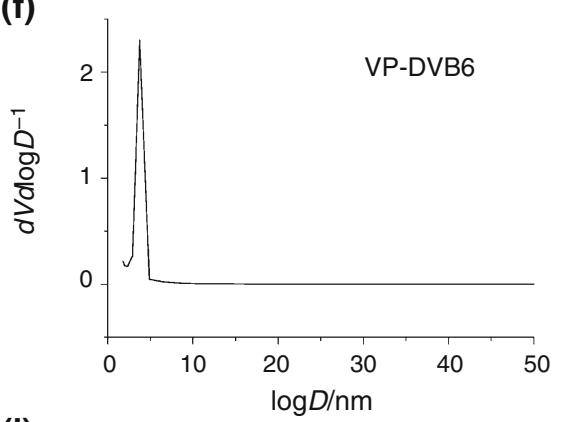

(i)

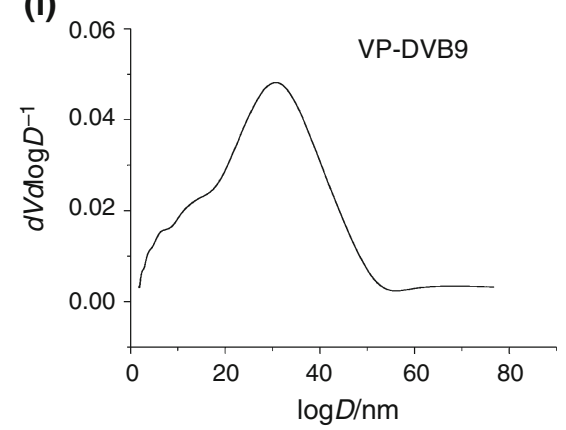

Fig. 8 Pore size distribution of VP-DVB copolymers

Figure 6 presents the internal structure of monolith synthesized in the presence of pure toluene. The individual nuclei responsible for high value of surface area and small pore diameter can be clearly seen. By contrast, monolith synthesized in pure decan-1-ol is displayed in Fig. 7. In this case, the nucleus lost their individuality, and huge cavities are observed inside the monolith.

The pore size distributions (PSD) were obtained from the desorption branch of the isotherm using the BarrettJoyner-Halenda $(\mathrm{BJH})$ procedure. The monoliths synthesized in the presence of pure toluene (VP-TRIM1 and VPDVB1) are characterized by narrow pore size distribution with the maximum at $4 \mathrm{~nm}$ (Figs. 8, 9). This fact indicates the late phase separation at greater monomer conversion. Addition of $15.5 \%(\mathrm{v} / \mathrm{v})$ of poor solvent (dodecane) results in shifting the $\mathrm{PSD}_{\max }$ toward larger pores. It is especially visible in the case of VP-TRIM 2 copolymer. The maximum of pore size distribution for this copolymer is observed at $390 \AA$ A. Further addition of dodecane has not so spectacular effects. In the case of VP-DVB copolymers, the effect is not so significant. Rate of phase separation is lower, and the pore size does not exceed the value of $25 \mathrm{~nm}$.

The effect of non-solvating co-solvent is also clearly visible in the case of decan-1-ol. Although addition of $1.4 \mathrm{~mL}$ of decan-1-ol does not change the position of $\mathrm{PSD}_{\max }$ comparing with monoliths obtained in the presence of pure toluene, further increase of the decan-1-ol volume results in increasing pore sizes. This effect is especially visible, when decan-1-ol starts to prevail in the solvents mixtures. In this case, phase separation occurs later. As a result, both large microglobules and large micropores are formed. A diminution of the solvating power of the porogenic mixtures allow to obtain copolymers with pores of great size. They approach macroporous region that is especially desirable in the synthesis of monoliths for HPLC purposes. 
(a)

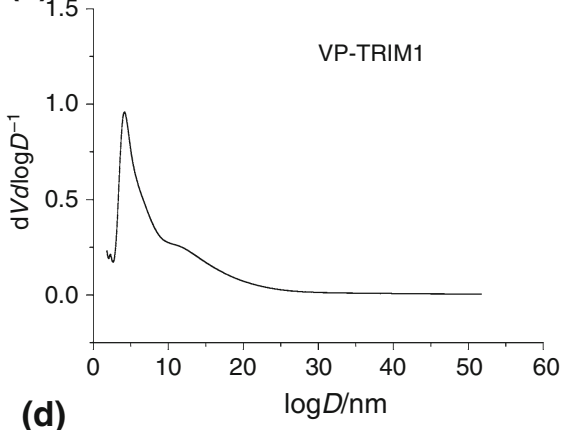

(d)
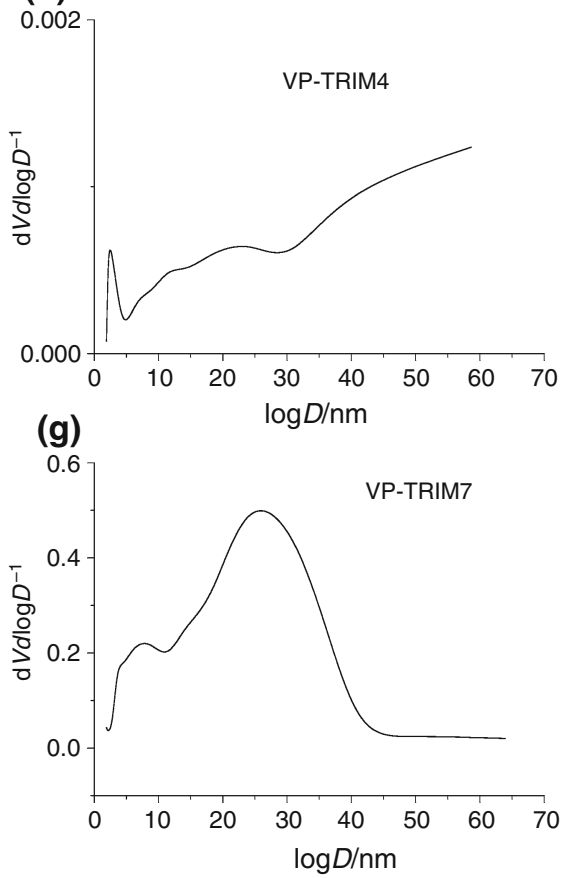

(b)
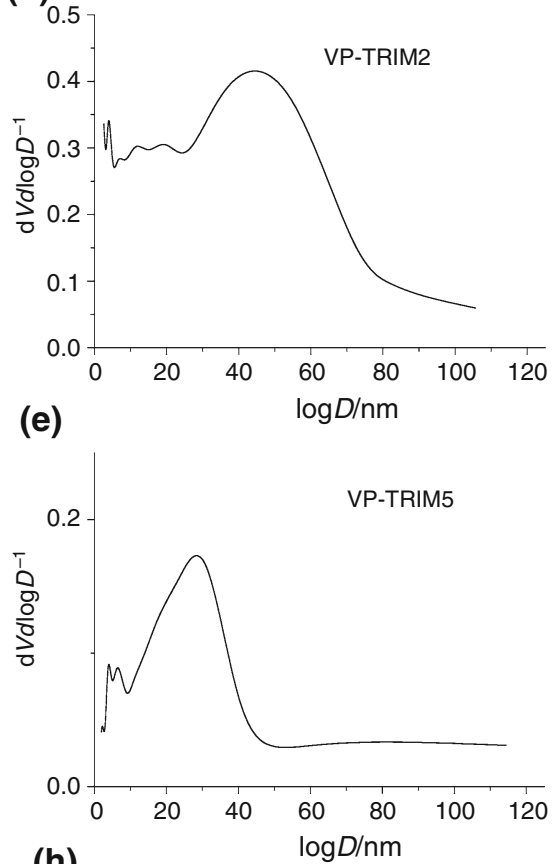

(h)

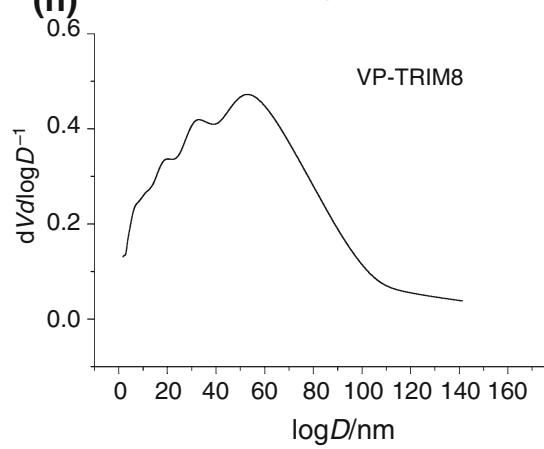

(c)

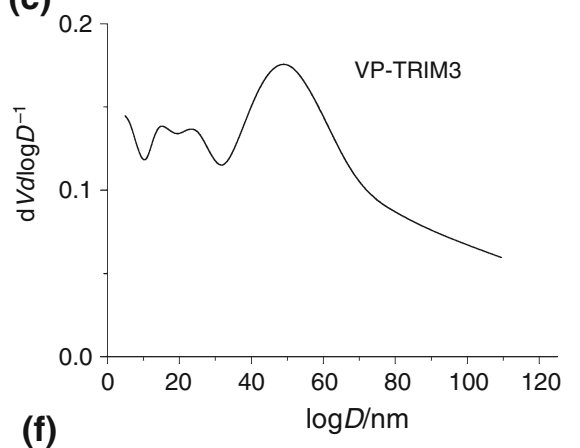

(f)

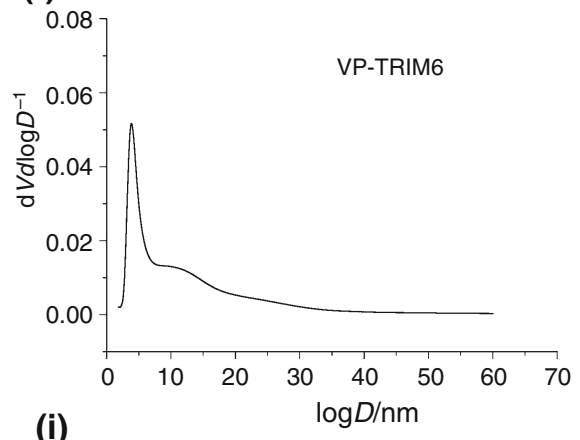

(i)

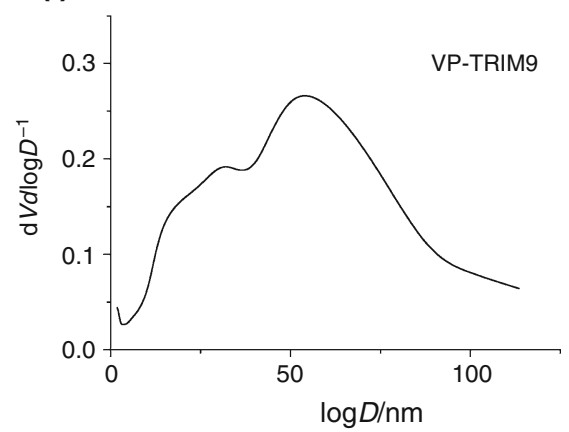

Fig. 9 Pore size distribution of VP-TRIM copolymers

Fig. 10 TG curves of VP-DVB (1) and VP-TRIM (2) copolymers

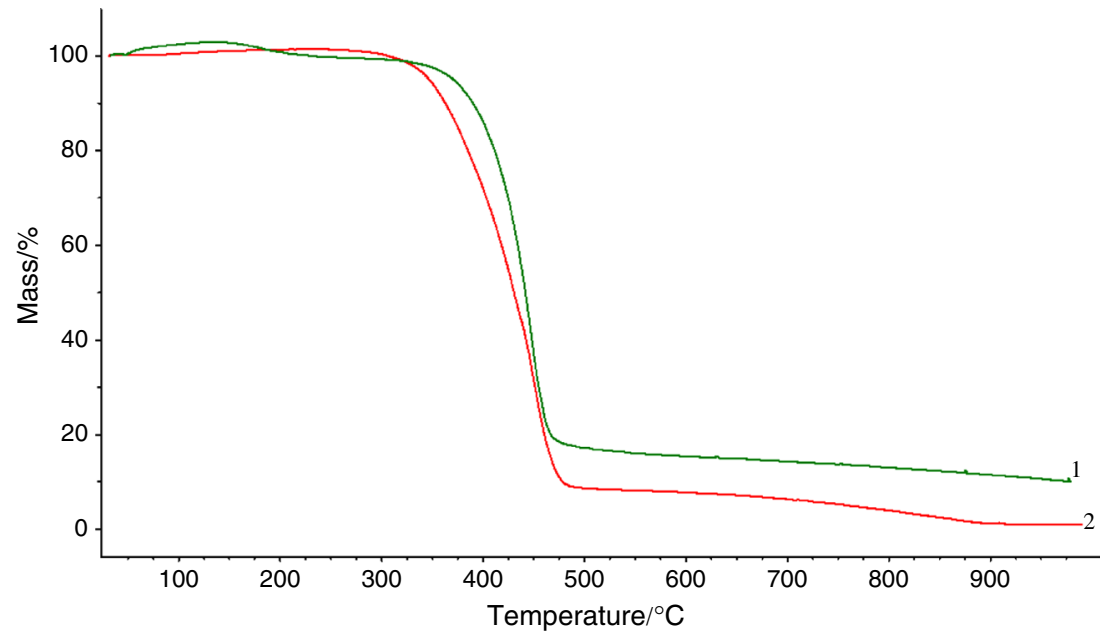

Another important task was to investigate the thermal properties of the obtained monoliths. The thermal behavior of all the samples was investigated by means of thermogravimetry (TG) and differential scanning calorimetry (DSC). TG curves obtained at the heating rate of $10 \mathrm{~K} \mathrm{~min}^{-1}$ in inert conditions are presented in Fig. 10. 
Fig. 11 DTG curves of VP-DVB (1) and VP-TRIM (2) copolymers

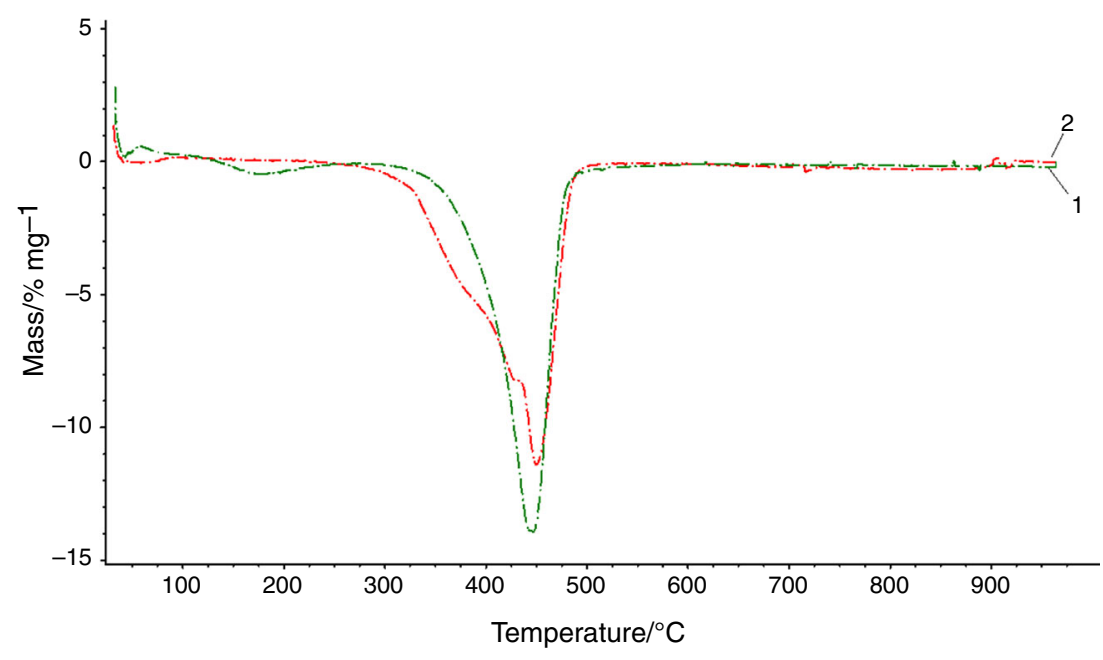

Table 3 Parameters evaluated from TG and DTG curves of the copolymers under study

\begin{tabular}{lllllll}
\hline Copolymer & $\mathrm{IDT} /{ }^{\circ} \mathrm{C}$ & $T_{20} \% /{ }^{\circ} \mathrm{C}$ & $T_{50} \%{ }^{\circ} \mathrm{C}$ & $\mathrm{FDT} /{ }^{\circ} \mathrm{C}$ & $T_{1 \max /}{ }^{\circ} \mathrm{C}$ & 447 \\
\hline VP-DVB & 369 & 412 & 442 & 540 & $T_{2 \max } /{ }^{\circ} \mathrm{C}$ \\
VP-TRIM & 345 & 386 & 431 & 482 & 430 & - \\
\hline
\end{tabular}

Fig. 12 DSC curves of VP-DVB (1) and VP-TRIM (2) copolymers

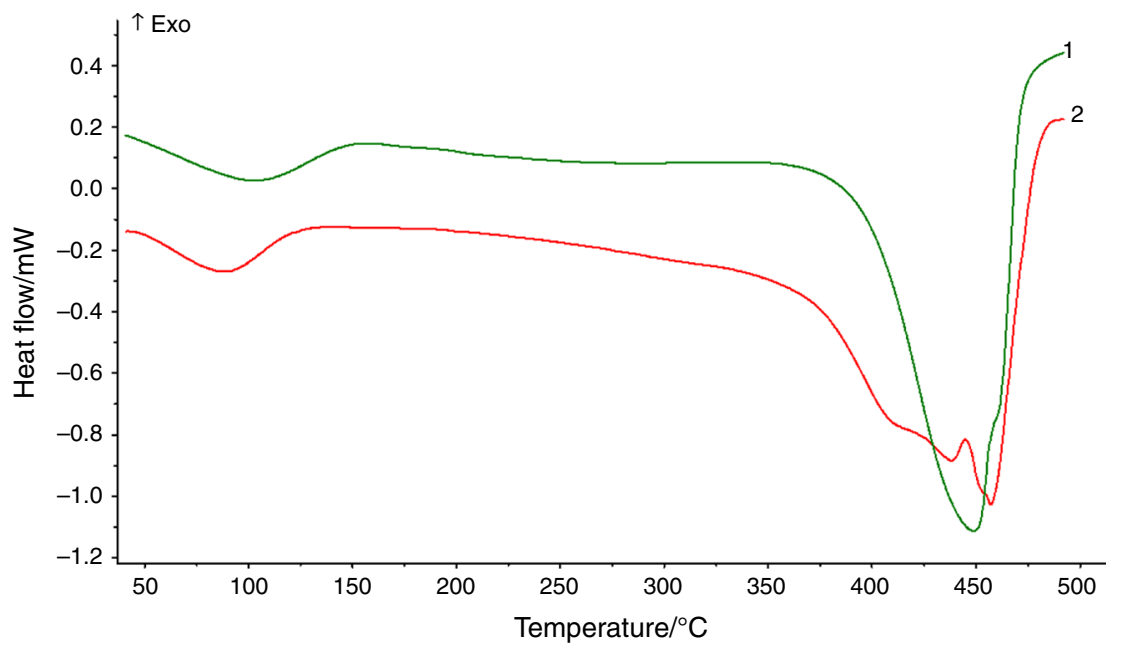

Figure 11 shows the DTG curves of the copolymer under study. The main parameters evaluated on the basis of these curves: the initial decomposition temperature (IDT), $T_{20} \%$ of mass loss, $T_{50} \%$ of mass loss, final decomposition temperature (FDT) and the temperature of the maximum of mass loss $\left(T_{\max }\right)$ are presented in Table 3. From this data, one can see that VP-DVB copolymer indicates higher thermal resistance than VP-TRIM. This phenomenon is directly connected with the chemical structure of the cross-linker used in the synthesis of the monoliths. The aromatic rings present in VP-DVB network causes that the process of thermal degradation starts in higher temperature comparing with VP-TRIM. What is more, the degradation process of VP-TRIM copolymers proceeds into two stages. The first with the maximum at $430{ }^{\circ} \mathrm{C}$ is connected with the process of breaking the ester bounds present in TRIM. The second with the maximum at $448{ }^{\circ} \mathrm{C}$ can be attributed to the degradation of the whole polymer network.

The differences between the studied copolymers were also confirmed during the DSC measurements. DSC curves determined in argon are presented in Fig. 12. 
Fig. 13 TG curves of VP-DVB copolymers obtained in the presence of $100 \%$ (1), $85 \%$ (2), $50 \%$ (3), $15 \%$ (4) and $0 \%$ toluene in the diluents mixture
Fig. 14 DTG curves of VPDVB copolymers obtained in the presence of $100 \%$ (1), $85 \%$ (2), $50 \%$ (3), $15 \%$ (4) and $0 \%$ toluene in the diluents mixture
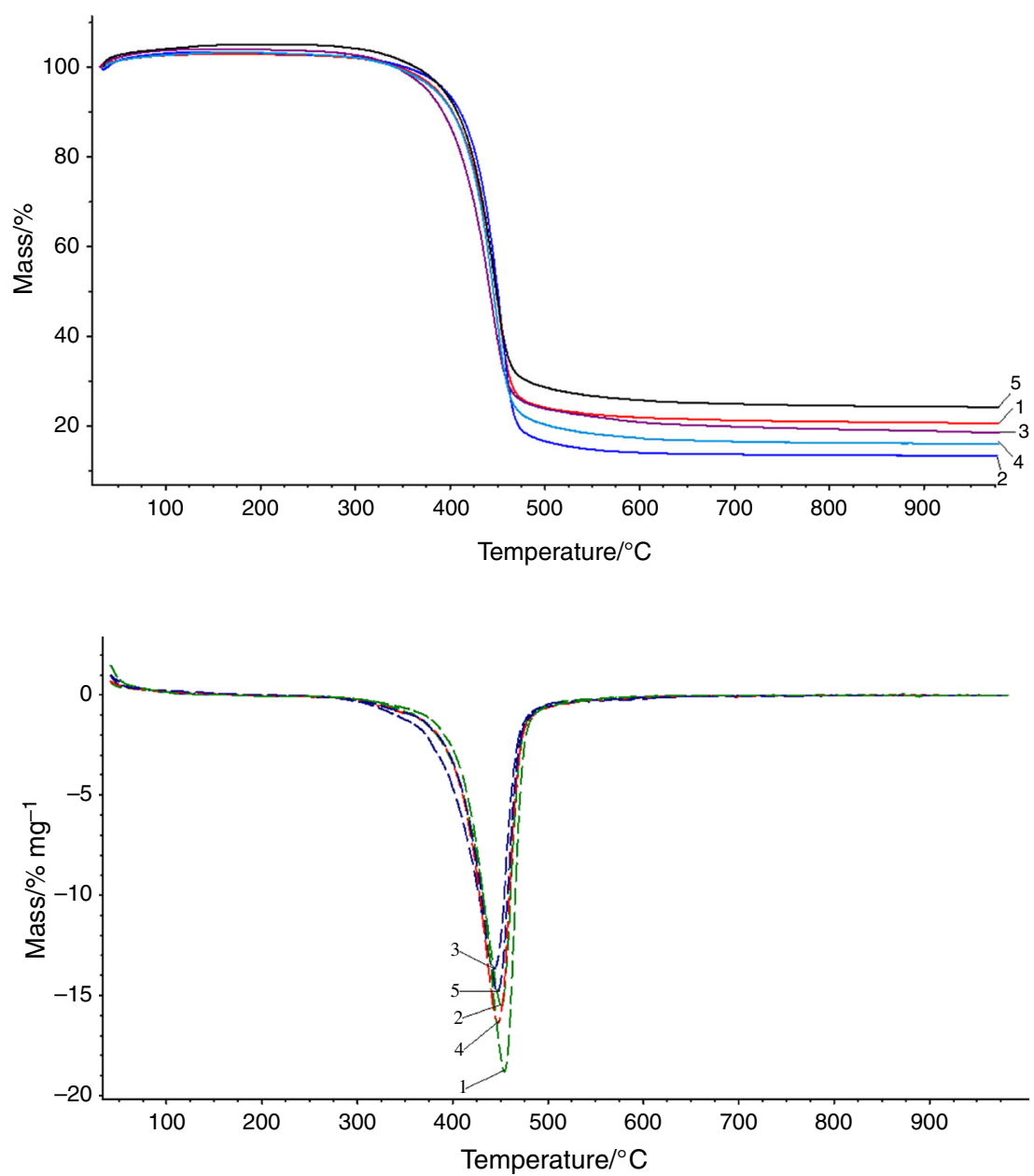

The DSC curves of the copolymers have characteristic, well-shaped calorimetric profile. Any exothermic peaks that could be attributed to the additional double bound conversion are not observed on the curves. As both copolymers have got highly developed porous structure and polar group present on the surface, they easily adsorb molecules of water present in the air. Consequently, despite the process of drying, some moisture is present in the polymer network. The first endotherimc peak with maximum at about $100{ }^{\circ} \mathrm{C}$ observed on both curves can be attributed to the process of water desorption.

The endothermic peaks observed at $400-500{ }^{\circ} \mathrm{C}$ correspond with the thermal degradation of copolymers. DSC measurement confirmed that the process of VP-TRIM degradation goes forward two stages. The maxima of the endotherimc peaks (438 and $457^{\circ} \mathrm{C}$ ) are in accordance with the values determined on the basis of DTG curves. In the case of VP-DVB copolymer, one peak with the maximum at $450{ }^{\circ} \mathrm{C}$ is observed in the discussed region.

Figures 13 and 14 present TG and DTG curves of VPDVB copolymers obtained with different diluent composition. As it was mentioned, solvating power of the diluent has enormous impact on the internal structure of the synthesized materials. No such effect was observed in the case of thermal properties. As can be seen in the figures, increasing the concentration of toluene in the mixture with n-dodecane has not change the position of the decomposition peaks.

\section{Conclusions}

The obtained experimental data clearly indicate that it is possible to control the porous properties of molded material within a broad range changing the diluent composition used during synthesis. It was noticed that monoliths prepared with only toluene as porogen had extremely small pores, which made them almost useless in flow-through applications. When only non-solvating diluent starts to prevail in the diluent mixture, materials with very low value of surface area are obtained. Porous properties can be readily adjusted by changing the ratio of non-solvating and solvating diluents. What is interesting, the diluent composition has no impact on the thermal properties. Thermal 
resistance of the copolymers strongly depends on the type of cross-linker used in the synthesis. The initial decomposition temperature of VP-DVB monoliths is $24{ }^{\circ} \mathrm{C}$ higher than their VP-TRIM counterparts.

Open Access This article is distributed under the terms of the Creative Commons Attribution License which permits any use, distribution, and reproduction in any medium, provided the original author(s) and the source are credited.

\section{References}

1. Gawdzik B, Maciejewska M. Synthesis of isobutyl maleatedivinylbenzene microspheres by different techniques of heterogeneous polymerizations. J Appl Polym Sci. 2004;91:2008-15.

2. Maciejewska M, Osypiuk J, Gawdzik B. Preparation and characterization of the chromatographic properties of ethylene glycol dimethacrylate/divinylbenzene polymeric microspheres. J Polym Sci A Polym Chem. 2005;43:3049-58.

3. Grochowicz M, Gawdzik B. Permanently porous copolymeric microspheres based on aromatic methacrylates. React Funct Polym. 2011;71:625-33.

4. Jaćkowska M, Bocian S, Gawdzik B, Grochowicz M, Buszewski B. Influence of chemical modification on the porous structure of polymeric adsorbents. Mater Chem Phys. 2011;130:644-50.

5. Grochowicz M, Bartnicki A, Gawdzik B. Preparation and characterization of porous polymeric microspheres obtained from multifunctional methacrylate monomers. J Polym Sci A Polym Chem. 2008;46:6165-74.

6. Drechny D, Trochimczuk AW. Synthesis and some sorptive properties of highly crosslinked cyanomethyl styrene/divinylbenzene copolymers. React Funct Polym. 2006;66:323-33.

7. Maciejewska M, Gawdzik B. Preparation and porous structure characterization of 4,4'-diphenylmethane dimethacrylate/divinylbenzene polymeric particles. J Appl Polym Sci. 2005;95:863-70.

8. Liu Q, Li Y, Shen S, Shanshan Z. The influence of crosslinking density on the pore morphology of copolymer beads prepared with a novel pore-forming agent. Mater Chem Phys. 2011;125:315-8.

9. Guiochon G. Monolithic columns in high-performance liquid chromatography. J Chromatogr A. 2007;1168:101-68.

10. Liu K, Aggarwal P, Lawson JS, Tolley HD, Lee ML. Organic monoliths for high-performance reversed-phase liquid chromatography. J Sep Sci. 2013;36:2767-81.

11. Buchmeiser MR. Polymeric monolithic materials: syntheses, properties, functionalization and applications. Polymer. 2007;48: 2187-98.
12. Maksimova E, Vlakh E, Sinitsyna E, Tennikova T. HPLC analysis of synthetic polymers on short monolithic columns. J Sep Sci. 2013;36:3741-9.

13. Healey R, Ghanem A. An insight to chiral monolith for enantioselective nano and micro HPLC: preparation and applications. Chirality. 2013;323:314-23.

14. Svec F. Preparation and HPLC applications of rigid macroporous organic polymer monoliths. J Sep Sci. 2004;27:747-66.

15. Müllner T, Zankel A, Mayrhofer C, Reingruber H, Höltzel A, Lv $\mathrm{Y}$, et al. Reconstruction and characterization of a polymer-based monolithic stationary phase using serial block-face scanning electron microscopy. Langmuir. 2012;28:16733-7.

16. Benes MJ, Horak D. Macroporous poly(vinylpyrrolidone-coethylene dimethacrylate) beads by suspension polymerization. React Funct Polym. 2000;45:189-95.

17. Zaidi SAR, Ali SW, Shah GB. Synthesis and characterization of a cation-exchange resin based on vinylpyrrolidone-co-divinylbenzene: effect of nature and amount of diluent on different resin properties. J Appl Polym Sci. 2004;92:3917-20.

18. Maciejewska M, Gawdzik J. Preparation and characterization of sorption properties of porous microspheres of 1-vinyl-2-pyrrolidone-divinylbenzene. J Liq Chromatogr Relat Technol. 2008; 31(7):950-61.

19. Maciejewska M. Characterization of macroporous 1-vinyl-2pyrrolidone copolymers obtained by suspension polymerization. J Appl Polym Sci. 2012;124:568-75.

20. Maciejewska M, Osypiuk-Tomasik J. Studies of sorption properties of porous copolymers of 1-vinyl-2-pyrrolidone. Therm Anal Calorim. 2013;111:1595-601.

21. Maciejewska M, Osypiuk-Tomasik J. Sorption on porous copolymers of 1-vinyl-2-pyrrolidone-divinylbenzene. Therm Anal Calorim. 2013;114:749-55.

22. Viklund C, Svec F, Frechet JMJ. Monolithic, "Molded", porous materials with high flow characteristics for separations, catalysis, or solid-phase chemistry: control of porous properties during polymerization. Chem Mater. 1996;8:744-50.

23. Svec F. Porous polymer monoliths: amazingly wide variety of techniques enabling their preparation. J Chromatogr A. 2010;1217:902-24.

24. Yu S, Ng FL, Ma KCC, Mon AA, Ng FL, Ng YY. Effect of porogenic solvent on the porous properties of polymer monoliths. J Appl Polym Sci. 2013;127:2641-7.

25. Brandrup J, Immergut EH, Grulke EA. Polymer handbook, vol 1. Wiley-Interscience, Hoboken. ISSN 0471-48171-8.

26. Sing K, Everett DH, Haul RA, Moscou L, Pierotti RA, Rouquerol J, Siemieniewska T. Reporting physisorption data for gas/solid systems with special reference to the determination of surface area and porosity. Pure Appl Chem. 1985;57:603-19. 